\title{
Pulmonary Tuberculosis in Children with HIV Infection: From Symptoms to Diagnosis and Treatment in a Pediatric Center in Yaoundé-Cameroon
}

\section{Abstract}

Introduction: Not only is tuberculosis common in sub-Saharan Africa, but also diagnostic barriers contributed to the delay of treatment in children. We described the diagnostic possibilities of tuberculosis in the context of HIV with limited resources.

Materials and Methods: A retrospective study was performed with the records of children co-infected with HIV-TB benefited from TB treatment in a pediatric center in Yaoundé. The time between the onset of symptoms, diagnosis and early treatment was assessed.

Results: A total of 18 files comprising all the clinical information were retained. Malnutrition (94.4\%), chronic cough (88.9\%) and prolonged fever $(44.4 \%)$ dominated the clinical picture. Between the onset of symptoms and the evocation of tuberculosis, we recorded at least two months in more than two thirds of patients. Only 11 (61.1\%) had received bacteriological research from their gastric secretions or sputum of whom 3 (27.3\%) showed the presence of bacilli. The diagnosis of the other 15 was made based on clinical assessment and in the absence of response to appropriate antibiotic therapy.

Conclusion: The initiation of antituberculous drugs to children co-infected with HIV was late. The delays in seeking care and financial barriers to paraclinical investigations have contributed. Strengthening the knowledge of health personnel would improve time-on treatment of tuberculosis whose diagnosis was done mostly without bacteriological evidence. The subvention of complementary tests would be a considerable contribution to our context.

Keywords: Co- infection; Tuberculosis; HIV; Child; Delay; Diagnosis

Received: February 04, 2016; Accepted: February 29, 2016; Published: March 06, 2016

\section{Introduction}

Tuberculosis (TB) worsens morbidity in children with HIV infection. The situation is a problem in Cameroon, where HIV prevalence among TB adults is about $30 \%$, and children account for $8.5 \%$ of people living with HIV [1,2]. The dual TB-HIV co-infection complicates the diagnosis of TB, it also provoke the delay of treatment $[3,4]$. The association of clinical and radiological signs, with a lack of response to antibiotic therapy would be optimal for the paraclinical workup when microscopy is negative [5].
Nguefack Félicitée ${ }^{1,2}$, Njiki Kinkela Mina Ntoto ${ }^{3}$, Dongmo Roger 4 , Chelo David ${ }^{1,3}$, Neh Flora ${ }^{5}$ and Koki Ndombo Paul Olivier ${ }^{1,3}$

1 Faculty of Medicine and Biomedical Sciences University of Yaounde I, Cameroon

2 Gynaeco-Obstetric and Pediatric Hospital of Yaoundé, Cameroon

3 Mother and Child Centre of the Chantal Biya Foundation, Cameroon

4 Institut supérieur de technologie médicale (ISTM), Yaoundé, Cameroon

5 Minitry of Public Health, Department of Family Health, Cameroon

Corresponding author: Nguefack Félicitée

Đ dongfel@yahoo.fr

Department of Pediatric, Faculty of Medicine and Biomedical Sciences, University of Yaoundé I, P.O. Box 1364, Yaoundé, Cameroon.

Tel: 237- 99591408

Citation: Félicitée N, Ntoto NKM, Roger D, et al. Pulmonary Tuberculosis in Children with HIV Infection: From Symptoms to Diagnosis and Treatment in a Pediatric Center in Yaoundé-Cameroon. J HIV Retrovirus. 2016, 2:1. 
can effectively diagnose tuberculosis, whether the patient is co-infected or not [5]. The most successful are expensive and thus inaccessible to all laboratories. Standard tests are used in our environment, although they are often partially realized. The culture of sputum or gastric fluid then would be the best investigation of pulmonary tuberculosis $[11,12]$. Before the advent of molecular diagnostic PCR, Ziehl Nielsen was the most efficient test. The aim of this study was to describe the diagnostic process, which will lead to the initiation of anti tuberculosis treatment in children co-infected with HIV.

\section{Materials and Methods}

A retrospective study examined the clinical records of a cohort of children infected with HIV and treated for pulmonary tuberculosis from January 2008 to December 2009 at the Mother and Child Centre of the Chantal Biya Foundation in Yaoundé. The anamnestic criteria, clinical and laboratory data which justified the prescription of anti-tuberculosis treatment were analyzed. We classified the patients with regards to high probability of tuberculosis according to the score of Crofton using these data [13]. For each patient, the time between the first symptoms, consultation and evocation the diagnosis of tuberculosis, HIV and initiation of TB and ARV was calculated. Anthropometric indices Weight/Height, Weight/Age and Height/Age defining global acute malnutrition, underweight and overall global chronic malnutrition were estimated according to WHO standards for children aged under 5 years [14]. In those older, nutritional status was calculated using the body mass index for age when the child had no edema [15]. Different indices were interpreted using the WHO-Anthro software Version 3.2.2. A child had a moderate malnutrition when the index value was between -3 and $-2 \mathrm{Z}$-score compared to the median of the reference population. It was termed severe when it was less than -3 Z-score and beyond -2 Z-score, nutritional status was said to be normal. Research of TB was done either in the gastric fluid in the young or in sputum when the children were at least 10 years. HIV diagnosis was done either by PCR in children under 18 months or by a serological test beyond this period.

\section{Ethical approval}

Our study was approved by the Ethics Committee of the Faculty of Medicine and Biomedical Sciences of the University of Yaoundé I.

\section{Statistical Analyses}

A descriptive analysis was performed with regard to categorical variables including sociodemographic parameters, clinical and laboratory data as well as the time-limits between different stages of management of subjects. These were expressed in frequencies or percentages. For continuous variables, the median or average were used with $95 \%$ confidence intervals. The values of the probability $\mathrm{P}<0.05$ were statistically significant.

\section{Results}

A total of 18 children who received TB treatment were included in the study. Both sexes were equally represented. The ages ranged from 5 months to 13 years, with a median of 6.2 years [IQR:
5.3 years]. The range of 5 to 9 years accounted for half of the subjects; there was no significant difference between age groups and gender $(p=0.324)$. Almost all patients had chronic cough and the majority (88.9\%) had been treated with different classes of antibiotics including ampicillin and gentamicin, or ceftiaxonegentamicin or cloxacillin-gentamicin and the macrolides or related drugs. Some had also received third-line antibiotics including quinolones, Amikacine, Imipenems. Only two subjects were already taking ARVs treatment.

Between the occurrence of symptoms and the evocation of TB diagnosis, it took at least 2 months in $77.8 \%$ of patients (Table 1). The time between the first symptoms and consultation in our site was already very long; more than two months in more than $3 / 4(77.8 \%)$ of subjects. Some $(27.8 \%)$ were taken spontaneously by parents (Table 1$)$. Others $(72.2 \%)$ were initially followed as outpatients before being hospitalized. The delay of consultation was not influenced by the mothers' age $\left(\mathrm{Khi}^{2}\right.$ = 7.79; $P=0.254)$. The notion of an index case of TB in the family was unknown in $13(72.2 \%)$ subjects. There was no difference between the time-limit of consultation and the initiation of the TB treatment $\left(\mathrm{Khi}^{2}=18.0 ; \mathrm{p}=0.249\right)$. The same was observed between the age of patient; the delay of consultation and also the delay of treatment $\left(\mathrm{Khi}^{2}=1.43 ; \mathrm{P}=0.8378\right.$ and $\mathrm{Khi}^{2}=27.6 ; \mathrm{p}=0.380$ respectively). Although not specified in half of patients, fever in the long term existed in nearly $44.4 \%$ (Table 2 ). Almost all (94.4\%) had a history of weight loss. According to the anthropometric indices, 9/18 (50.0\%) had severe acute malnutrition, $2 / 18(11.1 \%)$ had severe chronic malnutrition and 3/18 (16.7\%) severe underweight (Table 2). Chest radiography was performed in $17 / 18$ children $(94.4 \%)$ and the main lesions were diffuse parenchymal opacities $(52.9 \%)$ and reticulonodular (41.2\%) (Figure 1$)$. Only 8 patients $(44.4 \%)$ had received tuberculin skin test (IDR) with 4 positive. Search of TB was done in 11 patients (61.1\%) and was positive in $3(27.3 \%)$ specimens (Table 3 ). This examination was prescribed for all patients, however, most (54.8\%) more than three weeks after the initial consultation in our site. Similarly, between the date of the prescription of the examination and the date of its completion, there was a period of at least two weeks in those who had achieved. The result of TB search had not influenced the delay of treatment in children who performed the test $\left(\mathrm{Khi}^{2}\right.$ $=11.06 ; p=0.533$ ).

\section{Discussion}

The diagnosis of tuberculosis is difficult in the context of resource scarcity; HIV infection further complicates the situation. Despite the small sample size, this study had as aim to report the bottlenecks faced by the management of co-infection of HIV and TB. The therapeutic pathway before the diagnosis of tuberculosis in children with HIV and TB as well as criteria for anti-tuberculosis treatment was analyzed. Results show that the delay in the diagnosis of tuberculosis was a problem in the study. Between the first symptoms and diagnosis of certainty or presumption which justified the initiation of anti-TB treatment, it took more than two months in most subjects. Several reasons explain this situation first, 1) late resort for healthcare system. In Abidjan the time between the first symptoms and hospitalization was shorter, 
Table 1 Socio-demographic characteristics and time elapse between the various stages of patient management before treatment of tuberculosis.

\begin{tabular}{|c|c|c|}
\hline Variables & Frequency & $\%$ \\
\hline \multicolumn{3}{|l|}{ Place of residence } \\
\hline Urban & 16 & 88.9 \\
\hline Rural & 2 & 11.1 \\
\hline \multicolumn{3}{|l|}{ Age (Years) } \\
\hline$<5$ & 7 & 38.9 \\
\hline $5-9$ & 9 & 50.0 \\
\hline$\geq 10$ & 2 & 11.1 \\
\hline \multicolumn{3}{|c|}{ Time limit (days) between first symptoms - consultation $(n=18)$} \\
\hline$<30$ & 2 & 11.1 \\
\hline $30-59$ & 2 & 11.1 \\
\hline$>60$ & 14 & 77.8 \\
\hline \multicolumn{3}{|l|}{ Mode of admission in our site } \\
\hline Reference of a health facility & 13 & 72.2 \\
\hline Spontaneous & 5 & 27.8 \\
\hline \multicolumn{3}{|c|}{ Type of health facility referred to $(\mathrm{N}=13)$} \\
\hline Integrated health centre & 3 & 23.1 \\
\hline District hospital & 5 & 38.5 \\
\hline Clandestine health centres & 2 & 15.3 \\
\hline Private clinics & 3 & 23.1 \\
\hline
\end{tabular}

Time limit (days) between first symptoms - diagnosis of TB ( $n=18)$

$\begin{array}{lll}<30 & 0 & 0.0 \\ 30-59 & 4 & 22.2 \\ \geq 60 & 14 & 77.8\end{array}$

Time limit (days) between first symptoms -diagnosis of HIV ( $n=18)$

\begin{tabular}{l|l|l}
\hline Known HIV & 2 & 11.1 \\
\hline$<30$ & 2 & 11.1 \\
\hline $30-59$ & 3 & 16.7 \\
$\geq 60$ & 11 & 61.1
\end{tabular}

Time limit (days) between TB diagnosis- initiation of anti-TB treatment $(n=18)$

\begin{tabular}{|l|l|l|}
\hline$<7$ & 11 & 61.1 \\
\hline $7-14$ & 5 & 27.8 \\
\hline$>14$ & 2 & 11.1 \\
\hline
\end{tabular}

$\mathrm{TB}=$ Tuberculosis

standing at 12 days [16]. Some authors have demonstrated the long delay in diagnosis and attributed it to the parents [17]. Sensitization of communities for the recognition of symptoms, perception of the curability of the disease and the use of health services was crucial [18]. The problem also fall 2) on health personnel [19]. The time between the admission in hospital and 
Table 2 Distribution of patients according to the signs/symptoms of TB and rating score of Crofton and Miller.

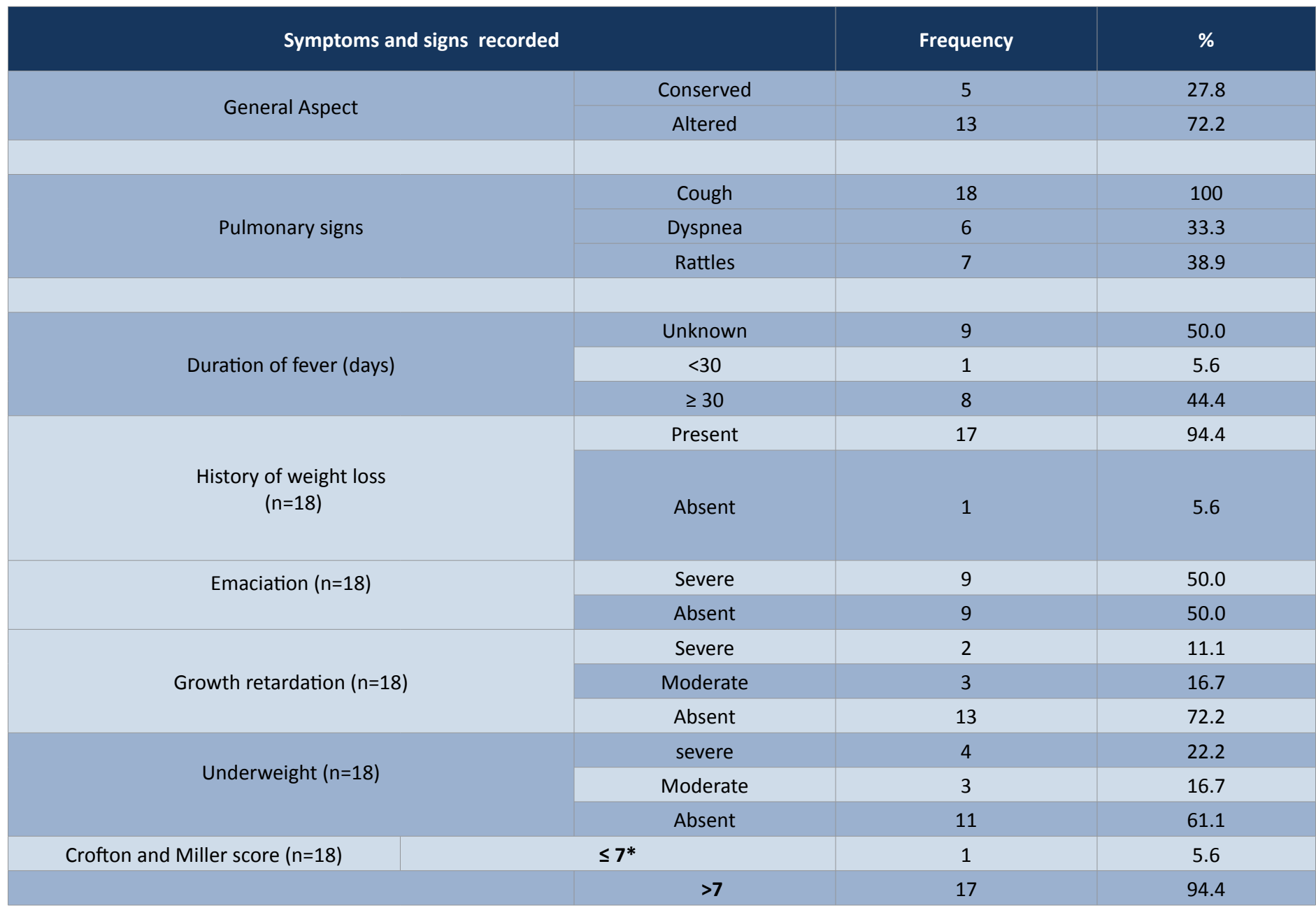

* Scores greater than 7 are in favor of a high likelihood of TB

Table 3 Distribution of patients according to the tuberculin test and search for tubercle bacillus.

\begin{tabular}{|c|c|c|c|}
\hline Variables & Modalities & Frequency & $\%$ \\
\hline \multirow{3}{*}{ IDR (Tuberculin Skin Test) } & Yes & 8 & 44.4 \\
\hline & No & 9 & 50 \\
\hline & Unknown & 1 & 5.6 \\
\hline \multirow{2}{*}{ IDR Results ( $n=8$ ) } & Positive & 4 & 50 \\
\hline & Negative & 4 & 50 \\
\hline \multirow{4}{*}{$\begin{array}{l}\text { AFB (Acid fast bacilli) in sputum or gastric } \\
\text { fluid ( } n=11)\end{array}$} & Yes & 11 & 61.1 \\
\hline & No & 7 & 38.9 \\
\hline & Presence of TB & 3 & 27.3 \\
\hline & Absence of TB & 8 & 72.7 \\
\hline
\end{tabular}

the evocation of the TB diagnosis was long in our site. Some staff would be poorly trained or they were focused on HIV instead of TB being investigated. In a rural area the inability of staff to diagnose partly explained the poor management of patients [20]. However, our population was mainly from an urban area (Table 1). The staff working in urban areas would also be less efficient. It would be a real problem of training of all health care givers on screening for tuberculosis. Indeed, chronic cough (Table 2) was often present $(100 \%)$, and a weight loss (94.4\%) associated in each case with a radio pulmonary disease (Figure $\mathbf{1}$ ). The radiological lesions were predominantly parenchymal opacities (52.9\%); also described in other studies [21]. Elsewhere, miliary and caverns were frequent in co-infected children [22]. These radiological signs would be more accurate than the symptoms and their association would increase diagnostic sensitivity [23]. Even in the context of HIV infection, all children should have received an IDR, a research for TB and the search for index cases. On another hand, we noticed 3 ) insufficient use of diagnostic facilities, in particular, the IDR and the search for TB, probably due to the high cost. In our site, simple tests such as IDR are not feasible at the bedside. Our results show 


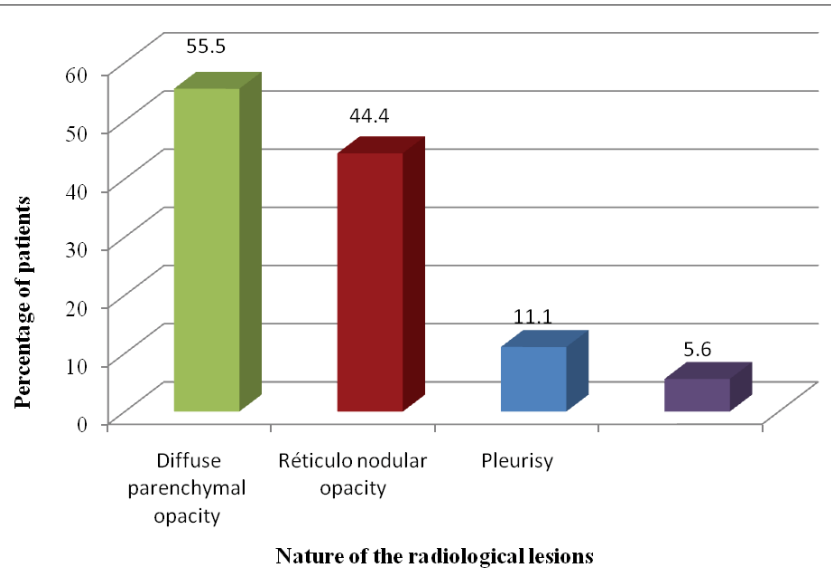

Figure 1 Pulmonary radiographic abnormalities.

that it was available in $44.4 \%$ of cases. In this site, patients should be taken to the Centre Pasteur laboratory of Cameroon close to our site in order to benefit at a price of US $\$ 5$. An exit permit is required for hospitalized subjects. Some parents would otherwise be unable to financially support these tests. Furthermore, the provision of care is done through out of pocket payments for families. Like many Cameroonians, most would live below the poverty line [24]. Thus the TB test was done in less than one third of subjects. To achieve a minimum of examinations, including X-ray, IDR and sputum test, parents must pay about US $\$ 42$. If we add the hospitalization costs, it will take more than $\$ 66$ US. In other contexts, only the radiological exams have increased the cost compared to routine investigations [25]. There would also exist 4) technical problems in the collection of specimen in young children. Also, parents would face difficulty retrieving results such as the culture of TB which is slow to arrive. However, the diagnosis of tuberculosis is based on anamnestic, clinical and paraclinical arguments [5-8]. On these elements are based rating systems which, although unreliable for therapeutic decision, are only an aid to screening $[9,26]$. One of the diagnostic criteria is the contact of the subject with a TB excretory adult $[27,28]$. It was not mentioned in most files that were reviewed. This notion was found in over $60 \%$ of patients in several studies $[16,29,30]$. Long-term fever existed only in $44.4 \%$ of patients. Some authors have excluded it from the definition criteria of tuberculosis in the community [31]. Severe weight loss was common among TB patients [32]. The analysis of anthropometric indices showed that whatever the type of malnutrition, they were not always altered in our study (Table 2). It is known that severe deterioration of weight should lead to investigation of HIV infection as well as co-infection with tuberculosis [33]. As in our study, the authors described a normal nutritional status in about $1 / 4$ of subjects [22]. The Weight/Age is one of the scoring elements for tuberculosis [34]. Although not recommended for TB diagnosis in a context of HIV, the use of system would nevertheless allow anticipation on investigations in order to shorten diagnostic time [9,35].

Ultimately, conventional methods of TB diagnosis are particularly insensitive to the direct examination of secretions. It is even more difficult in children because of the difficulty in obtaining sputum, and because they are less often bacillus excretory than adults. In HIV-TB co-infected children, diagnosis is even more complex because IDR is often negative, clinical signs are similar between these two diseases, which creates confusion and makes the system of scoring such as Crofton less specific.

We conclude that the delay of diagnosis of TB was long in our study and was due to several causes. Sensitization of parents on the signs, as well as retraining of staff would decrease the time of treatment initiation. New more sophisticated molecular biology techniques such as, PCR exist and would identify more effectively TB [36-38]. However, their extension would require a government subsidy because of their high cost. Free IDR, pulmonary X-ray and Research for TB especially in children infected with HIV is a measure that can reduce the diagnosis time. We suggest that a study carried out on a larger sample may provide more information that would be used to develop management algorithms of HIV-tuberculosis co-infection in our context. Nevertheless, the combination of chronic cough + weight loss + pathologic pulmonary Xray +/- long-term fever should be enough to make an IDR and research on TB, if children were referred earlier and the examinations prescribed systematically.

\section{Acknowledgments}

The authors thank the staff of the approved HIV treatment center of mother and child-CBF for their collaboration. They also thank Frédéric Tchouine for the statistical analyses. 


\section{References}

1 Ministère de la Santè Publique du Cameroun (2010) Directives nationales de prise en charge par les antirétroviraux des personnes (Adultes et Adolescents) infectées par le VIH. Yaoundè.

2 Ministère de la Santè Publique du Cameroun (2010) Plan stratègique national de lutte contre le VIH, le SIDA et les IST / 2011-2015. ComitèNational de Lutte contre le SIDA. Groupe Technique Central. Yaoundè.

3 Pefura Yone EW, Evouna Mbarga A, Kuaban C (2012) [The impact of HIV infection on childhood tuberculosis in Yaoundè, Cameroon. Rev Mal Respir 29: 1095-1103.

4 Venturini E, Turkova A, Chiappini E, Galli L, de Martino M, et al. (2014) Tuberculosis and HIV co-infection in children. BMC Infect Dis 14 Suppl 1: S5.

5 Huerga H, Varaine F, Okwaro E, Bastard M, Ardizzoni E, et al. (2012) Performance of the 2007 WHO algorithm to diagnose smearnegative pulmonary tuberculosis in a HIV prevalent setting. PLoS One 7: e51336.

6 Marais BJ, Gie RP, Hesseling AC, Schaaf HS, Lombard C, et al. (2006) A refined symptom-based approach to diagnose pulmonary tuberculosis in children. Pediatrics 118: e1350-1359.

7 Marais BJ, Gie RP, Obihara CC, Hesseling AC, Schaaf HS, et al. (2005) Well defined symptoms are of value in the diagnosis of childhood pulmonary tuberculosis. Arch Dis Child 90: 1162-1165.

8 Siddiqi K, Walley J, Khan MA, Shah K, Safdar N (2006) Clinical guidelines to diagnose smear-negative pulmonary tuberculosis in Pakistan, a country with low-HIV prevalence. Trop Med Int Health 11: 323-331.

9 WHO (2006) Guidance for National Tuberculosis Programmes on the management of tuberculosis in children: 1-41.

10 Osborne CM (1995) The challenge of diagnosing childhood tuberculosis in a developing country. Arch Dis Child 72: 369-374.

11 Oberhelman RA, Soto-Castellares G, Gilman RH, Caviedes L, Castillo $\mathrm{ME}$, et al. (2010) Diagnostic approaches for paediatric tuberculosis by use of different specimen types, culture methods, and PCR: a prospective case-control study. Lancet Infect Dis 10: 612-620.

12 Aminzadeh Z (2012) Evaluation of MODS Culture in the Diagnosis of Pulmonary Tuberculosis. Qom Univ Med Sci J 2: 21-27.

13 Lucas S (1992) Clinical tuberculosis J. Crofton, N. Horne \& F. Miller London \& Basingstoke: Macmillan Education, 1992. 210 pp. Price not stated. ISBN 0-333-56689-0 (paper-back). Trans R Soc Trop Med Hyg 86:702-703

14 OMS (2009) Normes de croissance OMS et identification de la malnutrition aiguë sévère chez l'enfant: 1-11.

15 WHO (2007) BMI-for-age (5-19 years).

16 Cisse L, Orega M, Niangue B, Plo K, Couitchere L, et al. (1999) Tuberculose et infection VIH de l'enfant hospitalisé a Abidjan: A propos de 56 cas. Médecine Afr Noire. 46:228-233.

17 Ayuo PO, Diero LO, Owino-Ong'or WD, Mwangi AW (2008) Causes of delay in diagnosis of pulmonary tuberculosis in patients attending a referral hospital in Western Kenya. East Afr Med J 85: 263-268.

18 Luis SF, Kamp N, Mitchell EM, Henriksen K, van Leth F (2011) Healthseeking norms for tuberculosis symptoms in southern Angola: implications for behaviour change communications. Int J Tuberc Lung Dis 15: 943-948.
19 Sendagire I, Schim Van der Loeff M, Mubiru M, Konde-Lule J, Cobelens F (2010) Long delays and missed opportunities in diagnosing smearpositive pulmonary tuberculosis in Kampala, Uganda: a crosssectional study. PLoS One 5: e14459.

20 Vellema SC, Durrheim DN, Smith JE (2008) Diagnosing childhood tuberculosis in rural clinics in Mpumalanga Province, South Africa. Curationis 31: 52-58.

21 Bolursaz MR, Khalilzadeh S, Baghaie N, Mehrian P, Ghafaripoor $\mathrm{H}$, et al. (2013) Clinical and Radiographic Findings of Pulmonary Tuberculosis in Infants. J Compr Pediatr 3:170-174.

22 Madhi SA, Huebner RE, Doedens L, Aduc T, Wesley D, et al. (2000) HIV-1 co-infection in children hospitalised with tuberculosis in South Africa. Int J Tuberc Lung Dis 4: 448-454.

23 Van't Hoog AH, Meme HK, Laserson KF, Agaya JA, Muchiri BG, et al. (2012) Screening strategies for tuberculosis prevalence surveys: the value of chest radiography and symptoms. PLoS One 7: e38691.

24 Cameroon:MDG Goal: Eradicate extreme poverty and hunger Health-related MDGs/fr - AHO.

25 van Cleeff MR, Kivihya-Ndugga LE, Meme H, Odhiambo JA, Klatser PR (2005) The role and performance of chest X-ray for the diagnosis of tuberculosis: a cost-effectiveness analysis in Nairobi, Kenya. BMC Infect Dis 5: 111.

26 Van Rheenen P (2002) The use of the paediatric tuberculosis score chart in an HIV-endemic area. Trop Med Int Health 7: 435-441.

27 Gulec SG, Telhan L, Koçkaya T, Erdem E, Bayraktar B, et al. (2012) Description of pediatric tuberculosis evaluated in a referral center in istanbul Turkey. Yonsei Med J 53: 1176-1182.

28 Hill P, Ota M (2010) Tuberculosis case-contact research in endemic tropical settings: design, conduct, and relevance to other infectious diseases. Lancet Infect Dis 10:723-732.

29 Sant'Anna CC, Santos MA, Franco R (2004) Diagnosis of pulmonary tuberculosis by score system in children and adolescents: a trial in a reference center in Bahia, Brazil. Braz J Infect Dis 8: 305-310.

30 Viani RM, Lopez G, Chacón-Cruz E, Hubbard P, Spector SA (2008) Poor outcome is associated with delayed tuberculosis diagnosis in HIV-infected children in Baja California, Mexico. Int J Tuberc Lung Dis 12: 411-416.

31 Gopi PG, Subramani R, Narayanan PR (2008) Evaluation of different types of chest symptoms for diagnosing pulmonary tuberculosis cases in community surveys. Indian J Tuberc 55: 116-121.

32 Pefura Yone EW, Kuaban C, Kengne AP (2012) HIV testing, HIV status and outcomes of treatment for tuberculosis in a major diagnosis and treatment centre in Yaounde, Cameroon: a retrospective cohort study. BMC Infect Dis 12: 190.

33 Okechukwu AA, Okechukwu OI (2011) Clinical correlate of tuberculosis in HIV co-infected children at the University of Abuja Teaching Hospital, Gwagwalada, Nigeria. Niger J Clin Pract 14: 206-211.

34 Harries A, Maher D, Raviglione M, Chaulet P, Nunn P (2004) Tuberculose et VIH: manuel clinique. Organisation mondiale de la santé (OMS):1-210.

35 Edwards DJ, Kitetele F, Van Rie A (2007) Agreement between clinical scoring systems used for the diagnosis of pediatric tuberculosis in the HIV era. Int J Tuberc Lung Dis 11: 263-269.

36 Connell TG, Zar HJ, Nicol MP (2011) Advances in the diagnosis of pulmonary tuberculosis in HIV-infected and HIV-uninfected children. J Infect Dis 204 Suppl 4: S1151-1158. 
37 Lima SS, Clemente WT, Palaci M, Rosa RV, Antunes CM, et al. (2008) Conventional and molecular techniques in the diagnosis of pulmonary tuberculosis: a comparative study. J Bras Pneumol 34: 1056-1062.
38 Zar HJ, Workman L, Isaacs W, Dheda K, Zemanay W, et al. (2013) Rapid diagnosis of pulmonary tuberculosis in African children in a primary care setting by use of Xpert MTB/RIF on respiratory specimens: a prospective study. Lancet Glob Health 1: e97-104. 